\title{
Chronic idiopathic myelofibrosis: independent prognostic importance of bone marrow microvascular density evaluated by CD105 (endoglin) immunostaining
}

\author{
Maurilio Ponzoni ${ }^{1}$, David G Savage ${ }^{2}$, Andrés JM Ferreri ${ }^{3}$, Giancarlo Pruneri ${ }^{4}$, \\ Giuseppe Viale ${ }^{4}$, Paolo Servida ${ }^{5}$, Francesco Bertolini ${ }^{6}$ and Attilio Orazi ${ }^{7}$ \\ ${ }^{1}$ Department of Pathology, San Raffaele H Scientific Institute, Milan, Italy; ${ }^{2}$ Division of Hematology- \\ Oncology, College of Physicians and Surgeons of Columbia University, New York, NY, USA; ${ }^{3}$ Department of \\ Radiochemotherapy, San Raffaele H Scientific Institute, Milan, Italy; ${ }^{4}$ Division of Pathology \& Laboratory \\ Medicine, European Institute of Oncology, Milan, Italy; ${ }^{5}$ Department of Hematology, San Raffaele H Scientific \\ Institute, Milan, Italy; ${ }^{6}$ Division of Hemato-Oncology, European Institute of Oncology, Milan, Italy and \\ ${ }^{7}$ Department of Pathology and Laboratory Medicine, Indiana University School of Medicine, Indianapolis, \\ IN, USA
}

\begin{abstract}
Microvascular density (MVD) is substantially increased in bone marrow biopsies of patients with chronic idiopathic myelofibrosis (CIMF). CD105, a useful molecule for assessing MVD in various malignancies, is preferentially expressed by recently formed microvessels. Increased serum-soluble CD105 in patients with chronic myeloproliferative disorders, including CIMF, was documented. CD105 MVD has not so far been investigated in CIMF: to this end, the results in 55 patients with CIMF and 21 controls were compared with the conventional CD34 immunostaining as well as traditional histological and clinical disease features. The MVD mean values estimated by both CD105 and CD34 were significantly higher in CIMF patients than in controls $(P<0.00001)$. In addition, the proportion of CD105-positive megakaryocytes was significantly higher in CIMF than in controls $(P<0.0001)$. A degree of reticulin fibrosis $>2$ correlated with increased CD105 MVD $(P=0.05)$. A multivariate analysis confirmed that CD105-positive MVD was an independent adverse prognosticator. This study demonstrates that while MVD, as assessed by both CD34 and CD105 immunostaining, is significantly increased in CIMF, only CD105-determined MVD correlates with the degree of fibrosis and is prognostically relevant. These findings provide a rationale for the investigational use of anti-CD105-targeted drugs in CIMF. Modern Pathology (2004) 17, 1513-1520, advance online publication, 23 July 2004; doi:10.1038/modpathol.3800224
\end{abstract}

Keywords: endoglin; CD105; bone marrow; myelofibrosis; prognosis

Chronic idiopathic myelofibrosis (CIMF) is a clonal myeloproliferative disease, which is characterized by mainly megakaryocytic and granulocytic proliferation, reactive deposition on bone marrow connective tissue and extramedullary hematopoiesis. ${ }^{1}$ The disease shows a progressive accumulation of fibrosis and an irreversible fatal course of variable length. The current therapy for CIMF is mostly palliative, although some limited success has been obtained by bone marrow transplantation. ${ }^{2,3}$ No

Correspondence: Dr M Ponzoni, MD, Department of Pathology, S Raffaele H Scientific Institute, Via Olgettina 60, 20132 Milano, Italy.

E-mail: ponzoni.maurilio@hsr.it

Received 13 January 2004; revised 10 June 2004; accepted 11 June 2004; published online 23 July 2004 clearcut prognostic factors have been identified so far.

Angiogenesis, which is characterized by the recruitment of new blood vessels, ${ }^{4}$ has been found to be crucial for the growth and proliferation of many solid tumors. There is evidence that, similar to other hematopoietic disorders, ${ }^{5,6}$ angiogenesis is involved in the pathogenesis and progression of CIMF. Serum levels of the proangiogenic cytokine VEGF and microvessel density (MVD) in the bone marrow are significantly increased in CIMF patients. ${ }^{7,8}$ Moreover, thalidomide, an antiangiogenic drug, is effective in combination with prednisone in counteracting cytopenia. $^{9}$ Finally, CD34-positive MVD has been reported to be an independent predictor of survival in CIMF patients. ${ }^{8}$ 
Angiogenesis has been most commonly assessed by immunostaining in routinely processed bioptic tissue with antibodies reactive with endothelial cells, including CD31, factor VIII, and CD34. In most studies, CD34 has been found to be the most useful antigen for assessing the number of microvessels (termed MVD) in both hematopoietic and nonhematopoietic malignancies.

The more recently introduced antiendoglin (CD105) monoclonal antibody appears to be superior to CD34 in the detection of new blood vessels in some solid tumors, such as non-small-cell lung carcinoma. ${ }^{10}$ Preliminary evidence has also shown that CD105-assessed MVD may be a better predictor of adverse outcome than CD34-assessed MVD in solid tumors, such as breast carcinoma. ${ }^{11}$ It has been recently demonstrated that serum levels of soluble CD105 are increased in patients with chronic myeloproliferative disorders, including one patient with CIMF. ${ }^{12}$

In the present study, we have compared for the first time the utility of CD105 and CD34 in the assessment of MVD in CIMF. We have correlated these measurements with known disease features such as degree of fibrosis and peripheral blood count values, as well as overall patient survival.

\section{Materials and methods}

\section{Patients}

The study population included 55 patients with CIMF. Patients with postpolycythemic or postthrombocythemic myelofibrosis and other myelofibrotic disorders were carefully excluded. There were 32 male and 23 female patients, with a median age of 72 (range 18-89) years. Peripheral blood values were available at diagnosis for 42 patients. In these subjects, the median hemoglobin, white cell and platelet counts were $11.5 \mathrm{~g} / \mathrm{dl}, 12.6 \times 10^{9} / \mathrm{l}$ and $448.5 \times 10^{9} / 1$, respectively. The control group consisted of 21 patients with bone marrow biopsies obtained for staging purposes for non-Hodgkin's lymphomas or for miscellaneous non-neoplastic conditions and found to be free of neoplasia and other abnormalities at histological examination. We deliberately did not include in the control group bone marrow biopsies taken from patients staged for Hodgkin's lymphoma, due to the increased microvascularity often seen in this setting (G Pruneri and $\mathrm{M}$ Ponzoni, personal communication).

\section{Pathologic Material and Immunohistochemistry}

Bone marrow biopsies (BMB) were collected from the Department of Pathology files of Columbia University, New York $(n=39)$ and $S$ Raffaele Hospital, Milan $(n=16)$, during the period 19902000 . Tissues were fixed with formalin $(n=17)$ or Bouin's solution $(n=38)$, decalcified with an EDTA- based solution or nitric acid, paraffin embedded and cut at $4 \mu \mathrm{m}$ thick sections. Control group included BMB fixed either with formalin or Bouin's solution. Each BMB was stained using hematoxylin and eosin and reticulin silver impregnation. Reticulin content was evaluated according to a scoring system previously published $;{ }^{13}$ cases were subdivided according to the degree of fibrosis ' $0-2$ ' and ' $3-4$ ', respectively. Immunohistochemistry was performed by the avidin-biotin peroxidase complex (ABC) method and the staining reaction revealed by $3,3^{\prime}-$ diaminobenzidine tetrahydrochloride chromogen method, using a DAKO automated immunostainer. For antigen retrieval, the slides were placed in a $0.01 \mathrm{M}$ EDTA buffer at $\mathrm{pH} 8$ and underwent three 4-min $780 \mathrm{~W}$ cycles at $90^{\circ}$ in a microwave oven before immunostaining.

\section{Bone Marrow Microvessel Staining and Estimation of MVD}

For CD105 and CD34 MVD assessment, 49 and 55 cases of CIMF were evaluable, respectively. Staining of microvessels was performed using anti-CD34 (QBEnd/10, Novocastra, Newcastle upon Tyne, UK; working dilution 1:100) and anti-CD105 (4G11, Novocastra; working dilution 1:400) monoclonal antibodies (MoAbs). The negative controls were similarly processed bone marrow biopsies in which nonimmune mouse serum was substituted for the primary antibody. For immunohistochemistry, proper internal controls were present in each sample and represented by blasts and arterioles for CD34 and by erythroid precursors and few sinusoids for CD105.

The measurement of MVD was performed according to a previously published method: ${ }^{14}$ any positively stained endothelial cell or endothelial cell cluster that was clearly separated from adjacent microvessels was considered as a single, countable microvessel. Vessel lumens were not a prerequisite to define a structure as microvessel. Sinusoid-like structures, as opposed to arterioles, were included in the definition of microvessel. Large vessels spanning two adjacent fields were counted only once. We modified our previous experience in visual microvessel scoring ${ }^{14}$ as follows: instead of choosing an arbitrary number of fields as previously published, all fields of the whole section were counted at $\times 200$ microscopic magnification and a final average MVD value for the whole section was registered. The described modification was introduced in order to avoid the bias of selecting areas with more abundant and/or more intensely stained microvessels. This is particularly important when dealing with CIMF, which is characterized both in early and in advanced stages by the presence of areas of greatly variable cellularity. In addition, in view of the CD105 reactivity with megakaryocytes (M Ponzoni and G Pruneri, unpublished observation), the presence of CD105-positive megakaryo- 
cytes was determined. The measurements were taken independently by two observers (MP and $\mathrm{AO}$ ), without the knowledge of any clinical data.

\section{Statistical Analysis}

Clinical characteristics and MVD among the study subgroups were compared using the $\chi^{2}$ tests or Fisher's exact test for categorical variables, according to the sample size. The comparison of mean values was performed using the 't-test'. Survival curves were generated by the Kaplan-Meier method. Overall survival (OS) was calculated from the date of the diagnosis to death or to the last date of followup. The prognostic relevance of CD34-positive and CD105-positive MVD was assessed using the mean MVD values in CIMF cases as a cutoff. Survival rates were reported as 5-year OS \pm standard error. Impact on survival of clinical and immunohistochemical variables were evaluated by comparing the survival curves by the log-rank test. The independent prognostic value of the variables was analyzed using the Cox model. All the probability values were twosided. Analyses were carried out using the Statistica 4.0 statistical package for Windows (Statsoft Inc., Tulsa, OK, USA).

\section{Results}

\section{MVD Assessed by CD105 and CD34 Immunostains}

The mean number of immunostained vessels per $\times 200$ field that were evaluated was 11 (range 4-22) and 14 (3-38) for controls and CIMF subgroups, respectively $(P=0.2)$. The mean $( \pm$ s.d.) MVD value for CD34 was $2.7 \pm 1.4$ (range 0.8-5.5) for controls and $37.7 \pm 24.7(6.3-100)$ for CIMF $(P<0.00001)$, respectively. The mean ( \pm s.d.) MVD value for CD105 was $8.2 \pm 2.8$ (range 2.5-14.1) in controls and $30.8 \pm 7.8$ (range 15.3-47.2) in CIMF $(P<0.00001)$. The mean MVD values for CD34 and CD105 immunoreactivity in CIMF cases were used as cutoffs to divide subgroups in correlation and survival analyses. There was no correlation between MVD calculated on the whole section and overall cellularity.

Both MoAbs highlighted the irregular shape of CIMF vessels, although CD105 was detected on a greater number of 'sinusoid-like' vessels ${ }^{5,14}$ than CD34 (Figure 1c,d). No difference in MVD was observed between formalin- and Bouin's solutionfixed specimens.

The percentage of cases with CD105-positive megakaryocytes was significantly increased in CIMF in comparison to the control biopsies: $36 / 49$ (73\%) vs $3 / 21(14 \%)$, respectively $(P<0.00001)$. In most cases, the megakaryocytes displayed weak-to-moderate cytoplasmic staining without predilection for scattered (rather than clustered) megakaryocytes (Figure 1e,f). No CD34-positive megakaryocytes were observed in the control group, while 2/35 $(6 \%)$ CIMF cases showed this finding $(P=0.29)$.

\section{MVD and Reticulin Fibrosis}

The control group did not show an increase in reticulin fibrosis. In the 50 valuable CIMF cases, reticulin content was ' $0-2$ ' in eight $(16 \%)$ cases and ' $3-4$ ' in $42(84 \%)$ cases. A significant correlation was observed between CD105-positive MVD and degree of fibrosis (ie degree 3-4) (mean \pm s.d. $=31.6 \pm 7.8)$ in comparison to a lesser degree of fibrosis (mean \pm s.d. $=25.7 \pm 7.5)(P=0.05)$. A significant correlation between CD105 megakaryocytes and substantial fibrosis was also observed; all cases with low degree of fibrosis (ie $\leq 2$ ) displayed CD105positive megakaryocytes, which were found in $57 \%$ of cases with an high degree (ie 3-4) of fibrosis $(P=0.05)$ (Table 1).

\section{Survival Analysis}

A total of 33 patients are alive at a median follow-up of 32 months (range 1-241), with a 5-year OS of $67 \pm 8 \%$. CD105-positive MVD (ie cases with CD105 MVD $\geq$ mean value, 30.8 MVD) was an adverse predictor of survival $(48 \pm 13 \%$ vs $86 \pm 8 \%, P=0.02)$ (Figure 2a and Table 2). CD34 MVD (Figure 2b), sex, fibrosis, hemoglobin, WBC and platelets values were not associated with poor survival.

Multivariate analysis, adjusted for the abovementioned parameters, confirmed the independent prognostic value of CD105-positive MVD for OS (Table 3).

\section{Discussion}

Previous research supports the view that tumor growth depends on angiogenesis. ${ }^{4}$ In addition to the well-established role of angiogenesis in solid tumors ${ }^{4}$ the increase in tumor vascularity has been previously investigated in a variety of hematological neoplasms, including acute lymphatic ${ }^{15}$ and myeloid $^{16,17}$ leukemias, myelodysplastic syndromes, ${ }^{14,18}$ Hodgkin's disease ${ }^{19}$ myeloma, ${ }^{20-25}$ lymphoproliferative disorders, ${ }^{26-31}$ systemic mastocytosis ${ }^{32}$ and chronic myeloproliferative disorders, ${ }^{5,14}$ including CIMF. ${ }^{8}$ The number of small vessels in a tumor, or MVD, is considered a surrogate indicator of angiogenesis. $^{4,22,24,33}$ The prognostic impact of MVD in hematological malignancies has been investigated by a number of groups, with conflicting results. ${ }^{15,16,20,21,23-25,30,31}$ In these studies, bone marrow microvessels are visualized by immunostaining of vascular endothelial cells; the stained microvessels are then counted and MVD determined. Commonly used antigens for assessment of MVD include CD34, CD31 and von Willebrand Factor (vWF). Of these, CD34 appears preferable to CD31 and vWF in 

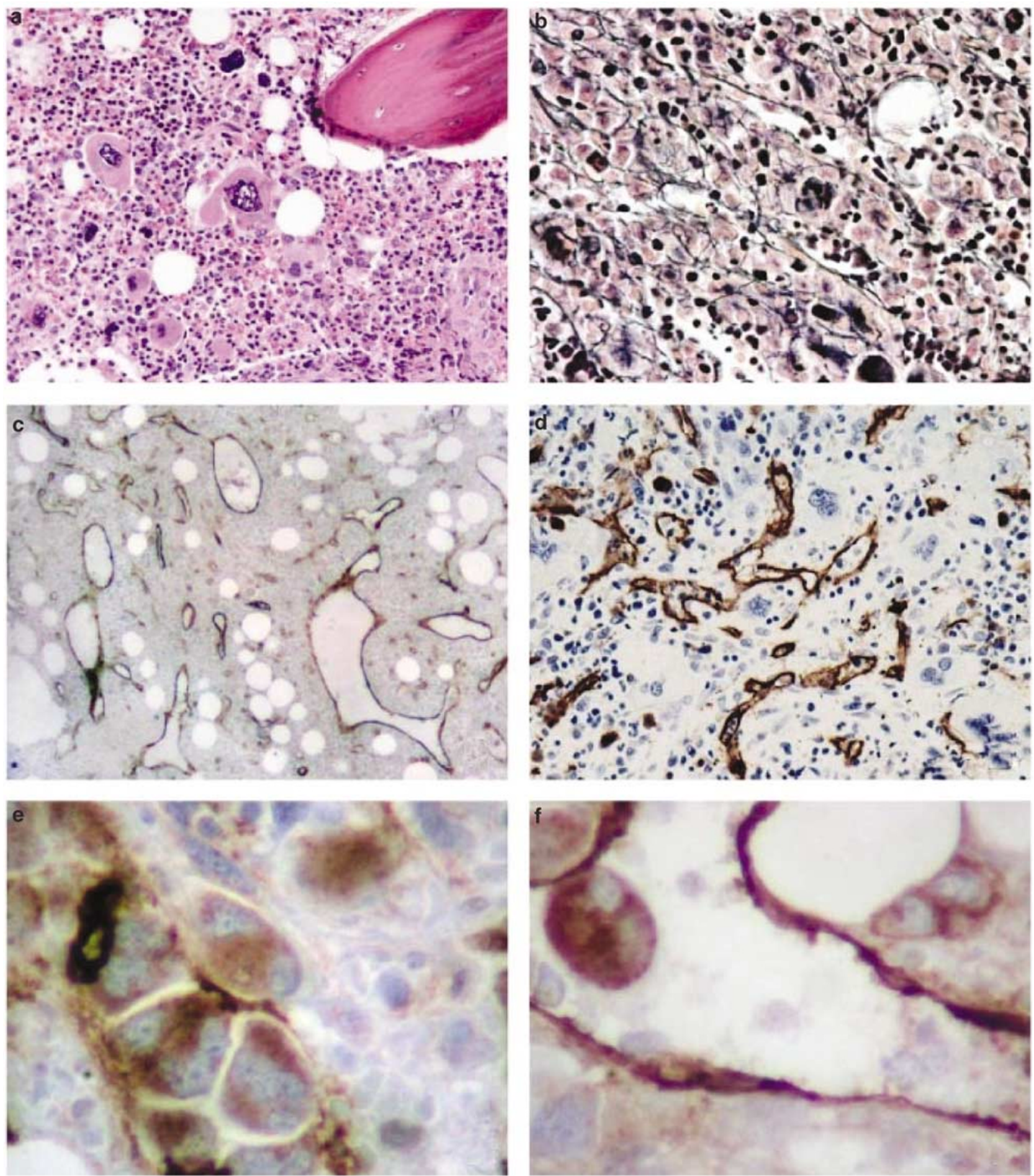

Figure 1 Increased MVD in chronic idiopathic myelofibrosis. A case of CIMF stained with hematoxylin and eosin (a), showing an increase in reticulin fibers when stained by silver impregnation (b); CD105 MoAb (c) preferentially highlights the 'sinusoid-like' morphology of increased microvessels when it is compared to CD34 immunostaining (d); clustered (e) and intrasinusoidal (f) megakaryocytes are immunoreactive for CD105.

patients with myeloid disorders because of its strong immunoreactivity with endothelial cells and its limited nonvascular expression. ${ }^{14,21,22}$ The value of CD34 for assessment of MVD in myeloproliferative disorders, including CIMF, has been demonstrated in recent studies. ${ }^{8,33}$

Our study shows that CD105 is also an effective tool for staining bone marrow microvessels and 
Table 1 Relationship between CD105 MVD, CD105-positive megakaryocytes and degree of fibrosis in CIMF

\begin{tabular}{lccc}
\hline Parameter & Fibrosis 1-2 & Fibrosis 3-4 & P-value \\
\hline CD105 MVD & $25.7 \pm 7.5$ & $31.6 \pm 7.8$ & 0.05 \\
CD105+ve meg & $8(100 \%)$ & $24(57 \%)$ & \\
CD105-ve meg & $0(0 \%)$ & $18(43 \%)$ & 0.05 \\
\hline
\end{tabular}

$\mathrm{MVD}=$ microvascular density $;$ meg $=$ megakaryocytes

a

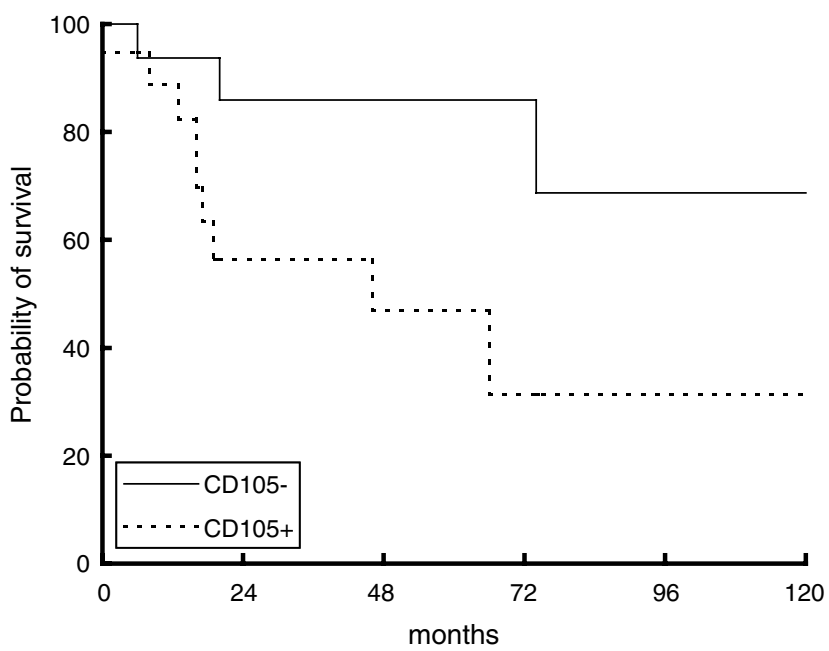

b

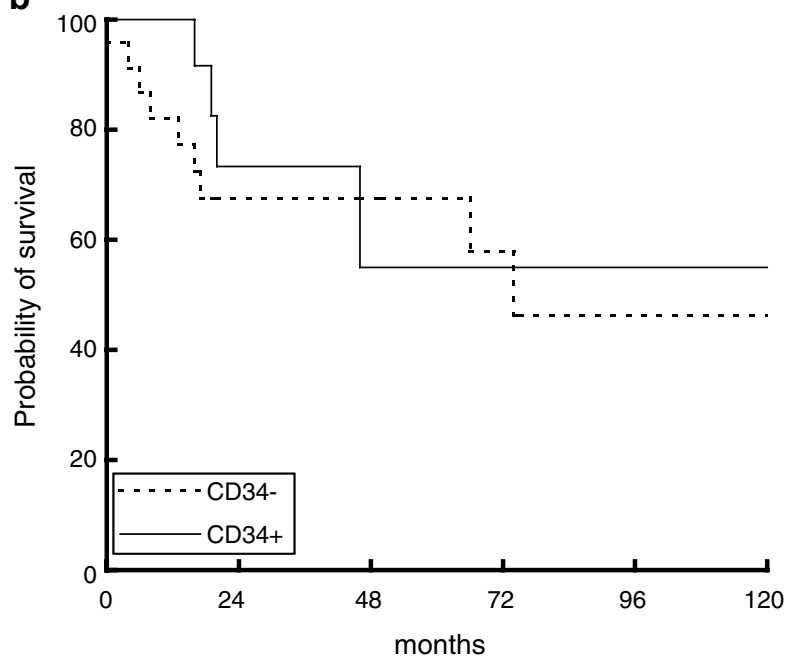

Figure 2 CD105-positive microvascular density is an adverse predictor of survival in chronic idiopathic myelofibrosis. Overall survival curves for patients grouped according to CD105 (a) and CD34 (b) immunoreactivity.

assessing angiogenesis in bone marrow biopsies of patients with CIMF. In these patients, similar to what has been observed for CD34, the density of CD105 stained microvessels is significantly higher than in the marrow of control patients. Although CD34 immunostaining can be used to assess MVD in
Table 2 Correlation between CD105 and CD34 MVD and overall survival in CIMF patients

\begin{tabular}{lllc}
\hline Parameter & Subgroup & 5-year OS \pm s.d. & P-value \\
\hline CD105 MVD & Negative vs positive & $86 \pm 8$ vs $48 \pm 13 \%$ & 0.02 \\
CD34 MVD & Negative vs positive & $68 \pm 10$ vs $55 \pm 18 \%$ & 0.22 \\
\hline
\end{tabular}

$\mathrm{MVD}=$ microvascular density; OS = overall survival. Cases were defined as positive for CD105 MVD when exhibited $\geq 30.8$ MVD and negative when $<30.8$ (mean value). Cases were defined as positive for CD34 MVD when exhibited $\geq 37.7$ MVD and negative when $<37.7$ (mean value). Sex, fibrosis, intravascular hemopoiesis, hemoglobin, white cell, and platelet counts did not correlate with survival.

Table 3 Multivariate analysis

\begin{tabular}{llcrc}
\hline Parameter & Subgroup & Odds ratio & 95\% CI & P-value \\
\hline CD105 MVD & Negative vs positive & 6.6 & $1.51-9.41$ & 0.02 \\
CD34 MVD & Negative vs positive & 0.4 & $0.11-8.03$ & 0.28 \\
Fibrosis & $1-2$ vs 3-4 & 1.4 & $0.17-2.53$ & 0.73 \\
Age & Continuous var. & 1.0 & $0.97-1.15$ & 0.20 \\
Hemoglobin & Continuous var. & 0.8 & $0.77-1.24$ & 0.11 \\
WBC & Continuous var. & 1.01 & $0.94-1.08$ & 0.81 \\
Platelets & Continuous var. & 0.9 & $0.9-1.01$ & 0.82
\end{tabular}

Cases were defined as positive for CD105 MVD when exhibited $>30.8 \mathrm{MVD}$ and negative when $<30.8$ (mean value). Cases were defined as positive for CD34 MVD when exhibited $\geq 37.7$ MVD and negative when <37.7 (mean value); var. = variable. CD105 MVD and CD34 MVD showed similar results when analyzed as continuous variables.

CIMF, ${ }^{8}$ our multivariate analysis results demonstrate that only CD105 can provide prognostically relevant information in this disorder (Figure 2). CD34-assessed MVD was not prognostically helpful (Table 3). Other parameters, including age, fibrosis, and peripheral blood values also lacked prognostic significance (Table 3).

The lack of prognostic significance for CD34 MVD values in CIMF are at variance with another recent study, in which increased MVD, as detected by CD34 (clone HPCA-1) immunostaining, was linked to a worse outcome. ${ }^{8}$ There may be several reasons for this discrepancy. Variations in immunostaining technique, such as the use of different primary antibodies among different laboratories, may be one factor. In contrast with Mesa et al, ${ }^{8}$ who employed the clone HPCA-1 for CD34 immunostaining, we used the QBEND/10 clone, which has been extensively utilized by our group and other investigators to assess MVD $^{14,16,24,34}$ In addition, in our study, a different approach for MVD evaluation was used. Instead of numbering semiquantitatively vessels as done by Mesa et al, ${ }^{8}$ we performed a complete count of all vessels in each section. ${ }^{24}$ This approach avoids the possible bias of selectively choosing areas with higher vascular density, and produces quantitative data without introducing predetermined arbitrary cutoffs or 'hot-spots'. It is noteworthy that the 
prognostic value of CD105 persisted when this parameter was analyzed as a continuous variable. Finally, Mesa et $a l^{8}$ included in their study a substantial number of patients with 'postpolycythemic' or 'post-thrombocythemic' myeloid metaplasia and performed multivariate analysis without adjusting for these separate clinicopathological entities.

The differences observed in our study may be due to the different endothelial specificity of the two antibodies. In contrast to CD105, which is preferentially expressed by recently formed vessels, ${ }^{11}$ CD34 is not a selective marker for newly formed vessels. Alternatively, our study might have failed to detect a prognostic role for CD34, at least in part, due to the bias intrinsic to retrospective investigations.

The significant correlation between CD105-assessed MVD and degree of fibrosis may not be fortuitous, since it has been shown that CD105, which interacts with receptors for transforming growth factor beta (TGF- $\beta$ ), is capable of modulating several of the cellular responses to this fibrogenetic cytokine. ${ }^{35,36}$ In CIMF, TGF- $\beta$ is strongly expressed by megakaryocytes, ${ }^{37,38}$ a large proportion of which express CD105.

Taken together, the fibrogenetic role for endoglin as well as its abnormally increased expression in megakaryocytes may be relevant to the currently accepted pathogenetic model for CIMF in which the megakaryocytes play a primary role by their production of fibrogenetic cytokines, which in turn affect the bone marrow microenvironment through autocrine or paracrine mechanisms. ${ }^{39-45}$

With regard to therapeutic aspects, clinical improvement offered by treatment with the antiangiogenetic drug thalidomide has been recently reported in CIMF, although the efficacy of this drug in reducing MVD is still uncertain. ${ }^{9,46}$ It is noteworthy that a recent preliminary report focused on CD105 suggested the efficacy of anti-CD105 MoAb therapy, in synergy with cyclophosphamide, in reducing tumor size in human skin/SCID (severe combined immunodeficiency) mouse chimeras bearing human breast cancer. ${ }^{47}$ CD105 may represent a target for antiangiogenic therapy; ${ }^{48}$ this belief is also supported by the concept that endothelial cells of tumor microvessels may be more susceptible to killing effects of anti-CD105 immunoconjugates than the vascular endothelium of normal tissues. ${ }^{49}$

The prognostically significant increase of CD105positive megakaryocytes in CIMF patients, in contrast to the absence in the marrows of lymphoproliferative disorders such as myeloma ${ }^{24}$ suggests a potential role for this MoAb as a target for abnormal cells of the megakaryocytic lineage. To confirm this hypothesis, CD105 expression needs to be studied in a wider range of myeloid disorders characterized by abnormal megakaryocytopoiesis, such as various leukemic conditions (eg acute megakaryoblastic leukemia) as well as in chronic myeloproliferative disorders and myelodysplastic syndromes.
In conclusion, our study shows that CD105assessment represents an improved method for MVD determination in CIMF. CD105 shares with CD34 the advantage of having fewer positive cells of nonendothelial nature, thus facilitating evaluation of MVD. CD105 is preferentially expressed by recently formed vessels, ${ }^{11,49}$ although nonspecific. ${ }^{49,50}$ The presence of CD105-positive MVD appears to worsen the prognosis of patients with CIMF. The potential therapeutic use of anti-CD105 immunotherapy in CIMF deserves investigation, particularly in the group of CIMF patients with highest CD105-MVD values.

\section{Acknowledgement}

We are grateful to Mr Stefano Grassi for excellent photographic editing assistance.

\section{References}

1 Thiele J, Pierre R, Imbert M, et al. Chronic idiopathic myelofibrosis. In: Jaffe ES, Harris NL, Stein $H$, Vardiman JM (eds). Tumors of Haematopoietic and Lymphoid Tissues. IARC Press: Lyon, France, 2001, pp 35-38.

2 Guardiola P, Anderson JE, Bandini G, et al. Allogeneic stem cell transplantation for agnogenic myeloid metaplasia: a European Group for Blood and Marrow transplantation, Societe Française de Greffe de Moelle, Gruppo Italiano per il Trapianto del Midollo Osseo, and Fred Hutchinson Cancer Research Center Collaborative Study. Blood 1999;93:2831-2838.

3 Anderson JE, Deeg HJ, Tefferi A, et al. Effective treatment of myelofibrosis (MF) by autologous peripheral blood stem cell (PBSC) transplantation (PBSCT). Blood 1999;94:396a (abstract).

4 Kerbel R, Folkman J. Clinical translation of angiogenesis inhibitors. Nat Rev Cancer 2002;2:727-739.

5 Lundberg LG, Lerner R, Sundelin P, et al. Bone marrow in polycythemia vera, chronic myelocytic leukemia and myelofibrosis has an increased vascularity. Am J Pathol 2000;157:15-19.

6 Bertolini F, Mancuso P, Gobbi A, et al. The thin red line: angiogenesis in normal and malignant hematopoiesis. Exp Hematol 2000;28:993-1000.

7 Di Raimondo F, Azzaro MP, Palumbo GA, et al. Elevated vascular endothelial growth factor (VEGF) serum levels in idiopathic myelofibrosis. Leukemia 2001;15:976-980.

8 Mesa RA, Hanson CA, Rajkumar SV, et al. Evaluation and clinical correlations of bone marrow angiogenesis in myelofibrosis with myeloid metaplasia. Blood 2000;96:3374-3380.

9 Mesa RA, Steensma DP, Pardanani A, et al. A phase II study trial of combination low-dose thalidomide and prednisone for the treatment of myelofibrosis with myeloid metaplasia. Blood 2003;101:2534-2541.

10 Tanaka FT, Otake Y, Yanagihara K, et al. Evaluation of angiogenesis in non-small cell lung cancer: comparison between anti-CD34 antibody and anti-CD105 antibody. Clin Cancer Res 2001;7:3410-3415. 
11 Kumar S, Ghellal A, Li C, et al. Breast carcinoma: vascular density determined using CD105 antibody correlates with tumor prognosis. Cancer Res 1999; 59:856-861.

12 Calabrò L, Fonsatti E, Bellomo G, et al. Differential levels of soluble endoglin (CD105) in myeloid malignancies. J Cell Physiol 2003;194:171-175.

13 Bauermeister DE. Quantification of bone marrow a-a normal range. Am J Clin Pathol 1971;56:24-31.

14 Pruneri G, Bertolini F, Soligo D, et al. Angiogenesis in myelodysplastic syndromes. Br J Cancer 1999;81: 1398-1401.

15 Perez-Atayde AR, Sallan SE, Tedrow U, et al. Spectrum of tumor angiogenesis in the bone marrow of children with acute lymphoblastic leukemia. Am J Pathol 1997;150:815-821.

16 Padrò T, Ruiz S, Bieker R, et al. Increased angiogenesis in the bone marrow of patients with acute myeloid leukemia. Blood 2000;95:2637-2644.

17 de Bont ES, Rosati S, Jacobs S, et al. Increased bone marrow vascularization in patients with acute myeloid leukemia: a possible role for vascular endothelial growth factor. Br J Haematol 2001;113: 296-304.

18 Korkolopoulou P, Apostolidou E, Pavlopoulos PM, et al. Prognostic evaluation of the micro vascular network in myelodysplastic syndromes. Leukemia 2001;15:1369-1376.

19 Doussis-Anagnostopoulou IA, Talks KL, Turley H, et al. Vascular endothelial growth factor (VEGF) is expressed by neoplastic Hodgkin-Reed-Sternberg cells in Hodgkin's disease. J Pathol 2002;197:677-683.

20 Munshi NC, Wilson C. Increased bone marrow micro vessel density in newly diagnosed multiple myeloma carries a poor prognosis. Semin Oncol 2001;28: 565-569.

21 Rajkumar SV, Mesa RA, Fonseca R, et al. Bone marrow angiogenesis in 400 patients with monoclonal gammopathy of undeterminate significance, multiple myeloma and primary amyloidosis. Clin Cancer Res 2002;8:2210-2216.

22 Sezer O, Niemoller K, Kaufmann O, et al. Decrease of bone marrow angiogenesis in myeloma patients achieving a remission after chemotherapy. Eur J Hematol 2001;66:238-244.

23 Kumar S, Fonseca R, Dispenzieri A, et al. Bone marrow angiogenesis in multiple myeloma: effect of therapy. $\mathrm{Br}$ J Haematol 2002;119:665-671.

24 Pruneri G, Ponzoni M, Ferreri AJ, et al. Micro vessel density, a surrogate marker of angiogenesis, is an independent predictor of survival in multiple myeloma patients. Br J Haematol 2002;118:817-820.

25 Choi JH, Ahn MJ, Jang SJ, et al. Absence of clinical prognostic value of vascular endothelial growth factor and micro vessel density in multiple myeloma. Int J Hematol 2002;76:460-464.

26 Vacca A, Ribatti D, Ruco L, et al. Angiogenesis extent and macrophage density increase simultaneously with pathological progression in B-cell non-Hodgkin's lymphomas. Br J Cancer 1999;79:965-970.

27 Kini AR, Kay NE, Peterson LC. Increased bone marrow angiogenesis in B cell chronic lymphocytic leukemia. Leukemia 2000;14:1414-1418.

28 Arias V, Soares FA. Vascular density (tumor angiogenesis) in non-Hodgkin's lymphomas and florid follicular hyperplasia: a morphometric study. Leukemia Lymphoma 2000;40:157-166.
29 Ridell B, Norrby K. Intratumoral vascular density in malignant lymphomas of B-cell origin. APMIS 2001;109:66-72.

30 Molica S, Vacca A, Ribatti D, et al. Prognostic value of enhanced bone marrow angiogenesis in early B-cell chronic lymphocytic leukemia. Blood 2002;100: 3344-3351.

31 Pazgal I, Zimra Y, Tzabar C, et al. Expression of basic fibroblastic growth factor is associated with poor outcome in non-Hodgkin's lymphomas. Br J Cancer 2002;86:1770-1775.

32 Wimazal F, Jordan JH, Sperr WR, et al. Increased angiogenesis in the bone marrow of patients with systemic mastocytosis. Am J Pathol 2002;160: 1639-1645.

33 Mesa RA, Hanson CA, Li CY, et al. Diagnostic and prognostic value of bone marrow angiogenesis and megakaryocyte c-Mpl expression in essential thrombocytemia. Blood 2002;99:4131-4137.

34 Orazi A, Shendrik I, Servida P, et al. Angiogenesis in agnogenic myeloid metaplasia. Mod Pathol 2001;14: $175 \mathrm{~A}$ (abstract).

35 Fonsatti E, Del Vecchio L, Altomonte $\mathrm{M}$, et al. Endoglin: an accessory component of the TGF-betabinding receptor-complex with diagnostic, prognostic, and bioimmunotherapeutic potential in human malignancies. J Cell Physiol 2001;188:1-7.

36 Guerrero-Esteo M, Sanchez-Elsner T, Letamendia A, et al. Extracellular and cytoplasmic domains of endoglin interact with the transforming growth factor-beta receptors I and II. J Biol Chem 2002;277: 29197-29209.

37 Martyre MC, Romquin N, Le Bousse-Kerdiles MC, et al. Transforming growth-factor $\beta$ and megacaryocytes in the pathogenesis of idiopathic myelofibrosis. $\mathrm{Br} J$ Haematol 1994;88:9-16.

38 Bock O, Schlu J, Reising D, et al. Expression of fibrogenic cytokines in bone marrow cells from chronic myeloproliferative disorders. J Clin Pathol 2002;55:10a (abstract).

39 Wickenhauser C, Hillienhof A, Jungheim K, et al. Detection and quantification of transforming growth factor and platelet-derived growth factor release by normal human megakaryocytes. Leukemia 1995;9: 13-15.

40 Schmitz B, Thiele J, Kaufmann R, et al. Megakaryocytes and fibroblast-interaction as determined in human bone marrow specimens. Leuk Res 1995;19: 629-637.

41 Michiel DF, Oppenheim JJ. Cytokines as positive and negative regulation of tumor formation and progression. Semin Cancer Biol 1992;3:3-15.

42 Pustzai L, Lewis CE, Lorenzen J, et al. Growth factors: regulation of normal and neoplastic growth. J Pathol 1993;169:191-201.

43 Schmitz B, Wickenhauser C, Thiele J, et al. Megakaryocyte induced fibroblast proliferation is enhanced by costimulation with IL-6/IL-3 and dependent on secretory and adhesion events. Leuk Res 1999;23: 723-729.

44 Thiele J, Kvaniscka HM, Boelthen B. Resident bone marrow macrophages in idiopathic (primary) myelofibrosis (IMF): a histochemical and morphometric study on sequential trephine biopsies. Leuk Res 1999;23: 983-985.

45 Chou JM, Li C-Y, Tefferi A. Bone marrow immunohistochemical studies of angiogenic cytokines and their 
receptors in myelofibrosis with myeloid metaplasia. Leuk Res 2003;27:499-504.

46 Piccaluga PP, Visani G, Pileri SA, et al. Clinical efficacy and antiangiogenic activity of thalidomide in myelofibrosis with myeloid metaplasia. A pilot study. Leukemia 2002;16:1609-1614.

47 Takahashi N, Haba A, Matsuno F, et al. Antiangiogenic therapy of established tumors in human skin/severe combined immunodeficiency mouse chimeras by antiendoglin (CD105) monoclonal antibodies, and synergy between anti-endoglin antibody and cyclophosphamide. Cancer Res 2001;61:7846-7854.

48 Fonsatti E, Altomonte M, Arslan P, et al. Endoglin (CD105): a target for anti-angiogenetic cancer therapy. Curr Drug Targets 2003;4:291-296.

49 Seon BK. Expression of endoglin (CD105) in tumor blood vessels. Int J Cancer 2002;99:310-311.

50 Balza E, Castellani P, Zijlstra A, et al. Lack of specificity of endoglin expression for tumor blood vessels. Int J Cancer 2001;94:579-585. 